\title{
Australian medical college fights accusations of misconduct in presidential election
}

In this item (BMJ 2016;355:i6626, doi:10.1136/bmj.i6626),

John Wilson is referred to a renal physician. He is in fact a

respiratory physician. We apologise for this error. 